\title{
Cases of domestic violence go unreported
}

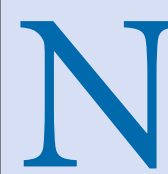

ew research from oral and maxillofacial surgeons (OMFS) at King's College Hospital has called for targeted training of frontline staff to improve recognition of domestic violence victims so appropriate safeguarding referral protocols are implemented.

The call from maxillofacial staff at King's follows the results of an 18-month study ${ }^{1}$ of all domestic violence cases with severe facial injuries treated by the King's OMFS team, and survey findings that looked at the attitudes of the maxillofacial team to recognising domestic violence. The move comes at a time when existing studies have found facial injuries reported in $76 \%$ to $94 \%$ of domestic violence cases, and make up $32 \%$ of all violent crimes.

The survey revealed frontline maxillofacial staff who treated domestic violence victims in the emergency department (ED) were only 'somewhat confident' in recognising victims of abuse. The accompanying case review confirmed that the majority of victims had been assaulted by current or ex-partners, and punched in the face or assaulted by household objects such as a knife or lamp.

Senior author, Consultant Maxillofacial Surgeon Kathy Fan, explained that because facial injuries are common in domestic violence cases that maxillofacial teams are ideally placed to screen for victims and alert safeguarding.

'We conducted the survey to find out how confident members of our team are at recognising victims of domestic violence, and looked at how many cases had been identified at King's over the past 18 months,' she said.

Co-author Holly Boyes, Maxillofacial junior trainee who works weekends and nights at King's, highlighted how a patient she had treated made her think that they should be picking up domestic violence cases at the time 'not afterwards when it's too late'.

In a recent incident, Boyes treated a patient who had had a bottle thrown at them in a bar, leaving the patient with a bad cut over an eye: 'I decided I should ask a little more about the injury, and that's when the patient started crying and admitted it had been their ex-partner who threw the bottle.

'The safeguarding team at King's is fantastic, so I asked for the patient's permission to ring them and made the referral. It seemed like a bit of a relief to the patient.'

She admitted that she hadn't been comfortable about asking, and realised that her colleagues would probably be in a similar position. She took the survey findings to the monthly maxillofacial team meeting and invited the King's safeguarding team to do some training about how to go about asking those difficult questions.

'If it's happening at King's, then maxillofacial teams everywhere are missing the signs of domestic violence. We're in the right place to offer the support of safeguarding teams to protect patients in the short and long-term,' she added.

While the case analysis revealed the majority of victims (13/18) had been punched, and assault with a common household object such as knife or lamp was involved in the other cases $(5 / 18)$ the team treated. The majority of alleged assailants $(10 / 18)$ were current partners or ex-partners (5/18).

The staff questionnaire found that oral and maxillofacial surgeons (23/35) were only 'somewhat confident' at recognising victims of domestic violence, although the majority (22/35) were aware the Trust had a domestic violence referral pathway. Asked whether they were confident to ask a patient about potential domestic abuse, half (18/35) said they were only 'somewhat confident' and 11 of the group were 'not so confident'. Only four of the respondents were 'extremely confident' in referring a patient to the Trust's domestic violence service.

The questionnaire concludes that targeted training for frontline maxillofacial staff is needed to increase confidence to identify and manage domestic violence patients and to refer them appropriately.

Fan added: 'Many cases are likely to have gone unidentified and unreported where injuries did not require treatment by the maxillofacial team.

'Victims with only minor facial injuries may have been treated by ED staff and discharged without referral to the maxillofacial team - and it's likely that patients would not have disclosed the true origin of their injury.'

\section{Reference}

1. Boyes $\mathrm{H}$ and Fan K. Maxillofacial injuries associated with domestic violence: experience at a major trauma centre. Br J Oral Maxillo Surg 2020; 58: 185-189.

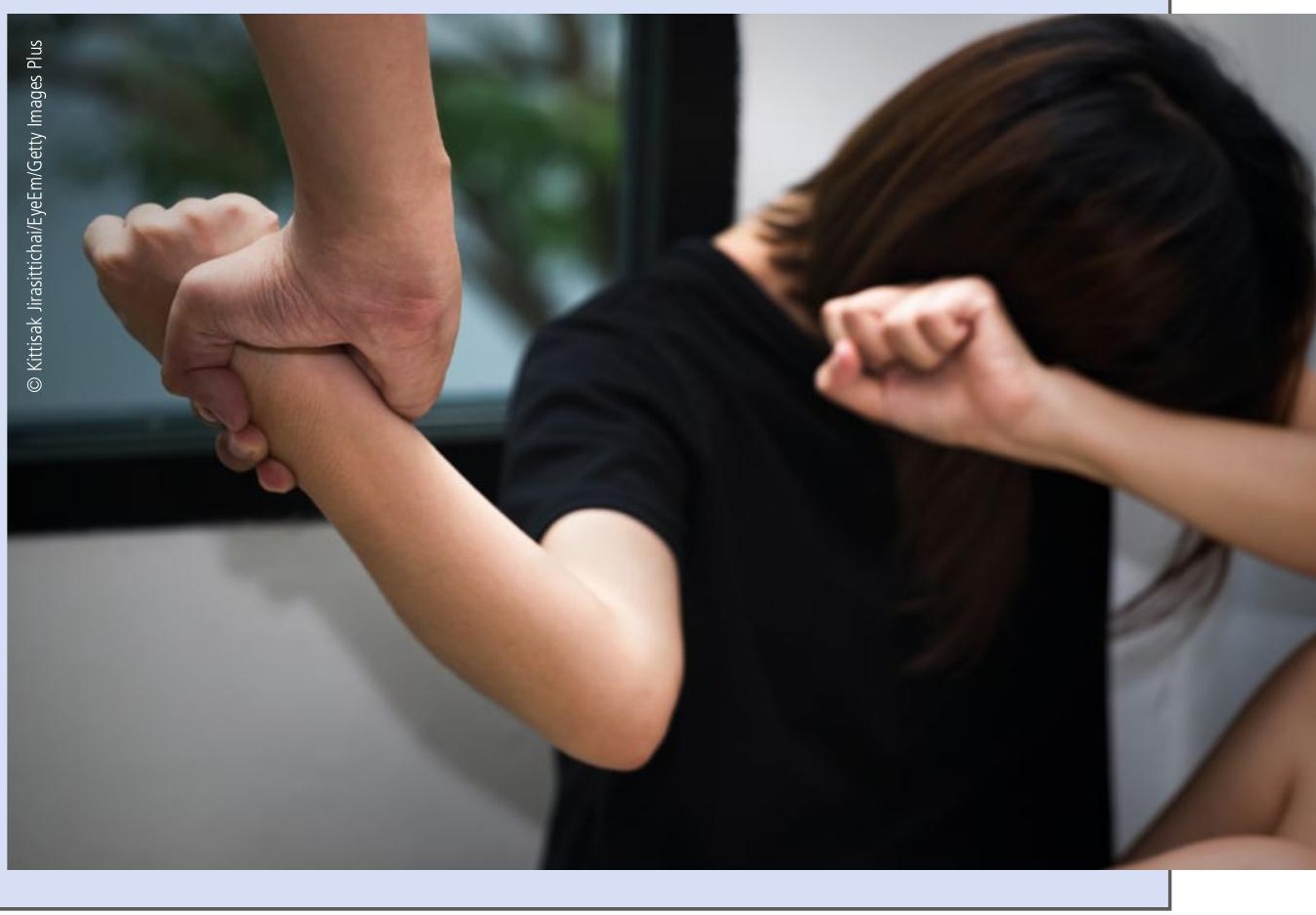

